\title{
Le corps microcosme, comme système de marquage zodiacal
}

Jean Richer

\section{(2) OpenEdition}

Journals

\section{Édition électronique}

URL : http://journals.openedition.org/shakespeare/1185

DOI : 10.4000/shakespeare.1185

ISSN : 2271-6424

Éditeur

Société Française Shakespeare

\section{Édition imprimée}

Date de publication : 1 novembre 1991

Pagination : 9-16

\section{Référence électronique}

Jean Richer, «Le corps microcosme, comme système de marquage zodiacal », Actes des congrès de la Société française Shakespeare [En ligne], 9 | 1991, mis en ligne le 01 janvier 2007, consulté le 24 avril 2019. URL : http://journals.openedition.org/shakespeare/1185; DOI : 10.4000/shakespeare.1185 


\section{SHAKESPEARE \\ ET LE CORPS A LA RENAISSANCE}

Société Française Shakespeare

Actes du Congrès 1990

sous la direction

de

M.T. JONES-DAVIES

PARIS

LES BELLES LETTRES

1991 
Tous droits de traduction, de reproduction et d'adaptation réservés pour tous les pays.

(C) 1991 Société d'édition Les Belles Lettres, 95 bd Raspail 75006 Paris

ISBN $2.25169117-\mathrm{X}$ 


\section{Le corps microcosme comme système de marquage zodiacal}

Je n'ai pas la prétention de traiter en une demi-heure un sujet aussi vaste et aussi important que celui du corps comme microcosme. Le titre exact de ma communication est plus restrictif : je parlerai du corps-microcosme comme système de marquage zodiacal dans le thêâtre de Shakespeare.

J'ai, depuis quelques années, entrepris d'établir que l'œuvre de Shakespeare est, pour une part considérable, construite selon des schémas astrologiques qui varient d'un ouvrage à l'autre ${ }^{1}$. C'est pourquoi chaque pièce doit faire l'objet d'une étude particulière, à ce point de vue. En ce qui conceme la répartition des personnages dans le zodiaque j'ai pu l'établir de manière détaillée dans dix ou douze cas déjà.

Elle se trouve indiquée de diverses manières, avec d'ailleurs de fréquents recoupements, par le nom même des personnages, par leur lieu d'habitation ou le nom de celui-ci, par des références à des animaux ou à des arbres; très souvent aussi Shakespeare décrit des images de l'Astrolabium planum de Johann Engel qui donne un répertoire des images des degrés du zodiaque. Cet ouvrage publié à Augsbourg en 1488 a une considérable importance pour l'origine des images employées par le dramaturge 2 . Le recours aux références aux vents et aux directions de l'espace devra faire l'objet d'une étude particulière.

En ce qui concerne le corps-microcosme, le système auquel le poète a recouru est le plus commun; il place la tête de l'homme dans le Bélier et les pieds dans les Poissons.

Dans un passage, souvent cité, qui se trouve au début de $L a$ nuit des rois (I. iii. 135-137) Shakespeare a fait une référence humoristique à l'homme-microcosme. Sir Toby dit à Sir Andrew :

" ... Ne naquîmes-nous pas dans le Taureau ?"

à quoi l'autre répond :

"Le Taureau, c'est les côtes et le cœur"

et Sir Toby reprend : 
"Non, Monsieur, c'est les jambes et les cuisses."

Comme le dit une note de l'édition Arden, Sir Andrew se trompe par ignorance et Sir Toby par malice, puisque le Taureau est ordinairement mis en relation avec le cou de l'homme. Mais ce qu'il faut surtout retenir, c'est qu'en 1601 un public londonien était assez familiarisé avec ce système de correspondances pour qu'il fût possible de susciter un effet comique en montrant sur la scène l'ignorance réelle ou supposée d'un personnage à son sujet. Il en résulte aussi qu'une partie au moins de ce public était capable de saisir au vol les multiples signaux de ce type qui jalonnent l'œuvre de Shakespeare.

Quand j'avais proposé mon sujet de communication, je pensais, du fait même de la multiplicité des procédés de marquage que j'ai indiquée, que je trouverai dans le théâtre de Shakespeare une sorte de saupoudrage de références astrologiques au corps humain, destinées à caractériser les personnages. Mais très vite, il m'est apparu que s'il existait bien quelques références isolées, là aussi, intervenait lunité de conception et de réalisation et que les références de ce type étaient groupées dans quelques pièces auxquelles elles contribuaient à donner leur tonalité particulière.

Le cas de Jules César (1599) est spécial, en ce sens que le nom même du personnage principal est une référence corporelle qui fait allusion à sa naissance "par césarienne". En outre, comme j'ai essayé de l'établir, les personnages principaux représentent des planètes désignées elles-mêmes par des métaux : or une découverte récente de la bio-chimie est que les sept métaux traditionnels, ainsi que quelques autres, sont présents dans le corps humain et en particulier dans la chaîne de l'A D N, où ils peuvent très bien être les récepteurs des influences planétaires, comme l'enseigne la tradition.

Dans Jules César, Brutus, tête du complot, est associé au signe du Bélier. Le personnage central, César, représente le Lion et le cœur de l'homme. Au troisième acte, s'adressant au cadavre de César, Antoine dit :

$O$ world, thou wast the forest to this hart;

And this indeed, o world, the heart of thee.

Tu étais, univers, la forêt de ce cerf,

(III. i. 207-208)

Et César, certes, ô monde, était ton cœur même. 
Cela propose une alternance entre le signe du Lion et le signe de la Balance, qui est, en réalité, la succession César Octave.

Le même jeu de mots se retrouve dans La nuit des rois à deux reprises (I. i. 17 et IV. i. 58).

L'appartenance zodiacale de Portia, femme de Brutus, est indiquée par le fait que, pour prouver son courage physique, elle se blesse volontairement à la cuisse (II. i. 300-301) : ce qui renvoie au Sagittaire, signe de feu. Si bien que dans la pièce, le triangle de feu est constitué par Brutus, César et Portia. Celle-ci se suicidera en avalant du feu : elle placera des braises dans sa bouche (IV. i. 155), ce qui est effectivement rapporté par Plutarque.

Enfin Casca représente une sorte de sous-Cassius et est associé au pied; j'en parlerai dans un instant.

Parmi les nombreuses significations astrologiques incluses dans les divers noms de Coriolan, il faut mentionner le radical cor, cordis qui renvoie au cour de l'homme et au signe du Lion qui caractérise le héros.

C'est ce même radical qui, dans Le roi Lear définit Cordelia et c'est bien l'excès de fierté qu'elle doit au Lion qui lui sera fatal. Lear représente le Bélier et la tête, cela est indiqué par son nom qui se rattache au radical lere et est confirmé par toutes les équivoques sur crown auxquelles se livre le Fou, par exemple lorsqu'il dit au vieux roi :

Tu n'avais guère de cervelle sous la couronne de ton crâne,

Le jour où tu t'es défait de ta couronne d'or.

(I.iv.169-170)

Kent est mis aux ceps sur l'ordre de Régane et de Cornouailles (II. ii. 150) pour avoir maltraité Oswald. Ce fait confirme son appartenance au Verseau qui gouverne les jambes. A bien des égards Kent est complémenetaire de Cordélia. Nous examinerons bientôt ce qui concerne le personnage de Cornouailles.

Je ne crois pas qu'il existe une pièce de Shakespeare dans laquelle douze personnages seraient caractérisés et situés par douze références corporelles. Mais, comme l'a bien établi James Dauphi$n e^{3}$, il en existe une où six personnages sont ainsi définis : c'est Cymbeline. Il s'agit de l'une des dernières pièces; on la date de 1609 et le système de références y est parfaitement au point. Six des personnages principaux s'y trouvent situés dans le zodiaque 
tant par des allusions aux organes et parties du corps que par leurs noms.

C'est ainsi que Iachimo remarque, en épiant Imogène, que celle-ci a sous le sein gauche: "Une fraise [... ] comme au cœur du coucou, cinq gouttes cramoisies". (trad. Maurice Castelain).

On her left breast

A mole cinque-spotted, like the crimson drops

I' the bottom of a cowslip.

(II. ii. $37-39)^{4}$

Cela revient à dire que le personnage appartient au signe du Cancer, signe lunaire qui gouverne la poitrine; cela est confirmé de diverses façons, par exemple, par le fait qu'Imogène rend à Diane un culte particulier. Son frère Guiderius, quant à lui, porte au cou

un signe remarquable, une étoile de sang

Guiderius had

Upon his neck a mole, a sanguine star

It is a mark of wonder

(V. v. 364-366)

Cela signifie que Guiderius est né sous le signe du Taureau, qui gouverne le cou.

Cymbeline, faisant l'éloge de Belarius, Guiderius et Arviragus déclare :

A vous qui de la Bretagne êtes le cœur, le foie et le cerveau, tout ce qui lui permet de vivre.

The liver, heart and brain of Britain

By whom I grant she lives.

(V. v. 14-15)

Belarius (Bel Aries), personnage conscient et réfléchi est la tête, régie par le signe du Bélier.

Dans la pièce le cour est l'attribut de Leonatus, le Lion, mais pourrait aussi convenir à Guiderius, futur coeur de la société. Le Capricorne, qui gouverne le foie, est le signe d'Arviragus dont le nom se décompose en (Hep)ar-vir-agus, c'est-à-dire foie-hommechèvre ce qui fournit une double identification. Au reste, au troisième acte (III. iii. 35-39) il médite sur le mois de décembre, période du Capricorne.

Le personnage de Cloten dont le nom, d'après l'étymologie clot ne peut signifier que "sticky" (gluant ou visqueux) est associé au signe des Poissons et aux pieds.

Dans le monologue de la scène $v$ de l'acte III ou entend Cloten méditer de violer Imogène et d'assassiner Leonatus Posthu- 
Cela propose une alternance entre le signe du Lion et le signe de la Balance, qui est, en réalité, la succession César Octave.

Le même jeu de mots se retrouve dans La nuit des rois à deux reprises (I. i. 17 et IV. i. 58).

L'appartenance zodiacale de Portia, femme de Brutus, est indiquée par le fait que, pour prouver son courage physique, elle se blesse volontairement à la cuisse (II. i. 300-301) : ce qui renvoie au Sagittaire, signe de feu. Si bien que dans la pièce, le triangle de feu est constitué par Brutus, César et Portia. Celle-ci se suicidera en avalant du feu : elle placera des braises dans sa bouche (IV. i. 155), ce qui est effectivement rapporté par Plutarque.

Enfin Casca représente une sorte de sous-Cassius et est associé au pied; j'en parlerai dans un instant.

Parmi les nombreuses significations astrologiques incluses dans les divers noms de Coriolan, il faut mentionner le radical cor, cordis qui renvoie au cœur de l'homme et au signe du Lion qui caractérise le héros.

C'est ce même radical qui, dans Le roi Lear définit Cordelia et c'est bien l'excès de fierté qu'elle doit au Lion qui lui sera fatal. Lear représente le Bélier et la tête, cela est indiqué par son nom qui se rattache au radical lere et est confirmé par toutes les équivoques sur crown auxquelles se livre le Fou, par exemple lorsqu'il dit au vieux roi :

Tu n'avais guère de cervelle sous la couronne de ton crâne,

Le jour où tu t'es défait de ta couronne d'or.

(I.iv.169-170)

Kent est mis aux ceps sur l'ordre de Régane et de Cornouailles (II. ii. 150) pour avoir maltraité Oswald. Ce fait confirme son appartenance au Verseau qui gouverne les jambes. A bien des égards Kent est complémenetaire de Cordélia. Nous examinerons bientôt ce qui concerne le personnage de Cornouailles.

Je ne crois pas qu'il existe une pièce de Shakespeare dans laquelle douze personnages seraient caractérisés et situés par douze références corporelles. Mais, comme l'a bien établi James Dauphi$n e^{3}$, il en existe une où six personnages sont ainsi définis : c'est Cymbeline. Il s'agit de l'une des dernières pièces; on la date de 1609 et le système de références y est parfaitement au point. Six des personnages principaux s'y trouvent situés dans le zodiaque 
tant par des allusions aux organes et parties du corps que par leurs noms.

C'est ainsi que Iachimo remarque, en épiant Imogène, que celle-ci a sous le sein gauche: "Une fraise [... ] comme au cour du coucou, cinq gouttes cramoisies". (trad. Maurice Castelain).

On her left breast

A mole cinque-spotted, like the crimson drops

I' the bottom of a cowslip.

(II. ii. $37-39)^{4}$

Cela revient à dire que le personnage appartient au signe du Cancer, signe lunaire qui gouverne la poitrine; cela est confirmé de diverses façons, par exemple, par le fait qu'Imogène rend à Diane un culte particulier. Son frère Guiderius, quant à lui, porte au cou

un signe remarquable, une étoile de sang

Guiderius had

Upon his neck a mole, a sanguine star

It is a mark of wonder

(V. v. 364-366)

Cela signifie que Guiderius est né sous le signe du Taureau, qui gouverne le cou.

Cymbeline, faisant l'éloge de Belarius, Guiderius et Arviragus déclare :

A vous qui de la Bretagne êtes le cœur, le foie et le cerveau, tout ce qui lui permet de vivre.

The liver, heart and brain of Britain

By whom I grant she lives.

Belarius (Bel Aries), personnage conscient et réfléchi est la tête, régie par le signe du Bélier.

Dans la pièce le cour est l'attribut de Leonatus, le Lion, mais pourrait aussi convenir à Guiderius, futur cœur de la société. Le Capricorne, qui gouverne le foie, est le signe d'Arviragus dont le nom se décompose en (Hep)ar-vir-agus, c'est-à-dire foie-hommechèvre ce qui fournit une double identification. Au reste, au troisième acte (III. iii. 35-39) il médite sur le mois de décembre, période du Capricome.

Le personnage de Cloten dont le nom, d'après l'étymologie clot ne peut signifier que "sticky" (gluant ou visqueux) est associé au signe des Poissons et aux pieds.

Dans le monologue de la scène $v$ de l'acte III ou entend Cloten méditer de violer Imogène et d'assassiner Leonatus Posthu- 
mus; le personnage y apparaît dans toute sa turpitude et son projet n'est pas sans analogie avec les intentions de Caliban à l'égard de Miranda. Il dit en conclusion : when my lust hath dined [...] I'll knock her back, foot her home again

(III. v. 145)

L'emploi du verbe to foot pour to kick, en cet endroit, est particulièrement révélateur. Il existe un autre emploi de ce mot comme verbe dans le Marchand de Venise (I. iii. 119), en un endroit où Shylock se compare à un chien que l'on chasse à coups de pied:

And foot me as you spurn a stranger cur.

Shakespeare a pour les natifs des signes d'eau: Cancer, Scorpion et Poissons, un mépris qui ne peut se justifier rationnellement, car c'est une simple question de probabilité, ces trois signes représentent le quart de la durée de l'année solaire et il existe de nombreux êtres humains fort acceptables marqués par ces mois. Mais ce sont surtout les natifs des Poissons que le dramaturge voue à l'exécration et bien que j'aie renoncé à passer en revue toutes les parties du corps, je crois intéressant d'insister un peu sur la relation des Poissons avec les pieds, telle qu'elle apparaît constamment chez l'auteur qui nous occupe. C'est une assez étonnante brochette de coquins, de misérables et de sous-hommes que ces Poissons qui défilent dans le thêâtre de Shakespeare. Casca dans Jules César, Cornouailles dans Le roi Lear, Caliban dans $L a$ tempête. Le nom même de Casca veut dire "cas-caco", "vain et méchant" et il est de manière répétée qualifié de dull ou blunt. Dans un curieux passage il s'identifie à son pied :

And I will set this foot of mine as far

As goes farthest

(I. iii. 117-120)

Dans le Roi Lear, le vil et cruel Cornouailles appartient aussi aux Poissons et son nom donne au Fou l'occasion d'équivoquer sur cor au pied et couronne - le cor aspirant à la couronne comme Caliban à la royauté de lîle enchantée. Voici un passage d'une chanson du Fou :

The man that makes his toe

What he his heart should make,

Shall of a corn cry woe,

And turn his sleep to wake.

(III. ii. 31-36)

Dans ces vers toe et corn renvoient à Cornwall et heart à Cordélie. 
La fable des membres et de l'estomac que propose Menenius Agrippa à la plèbe dès le début de Coriolan (I, i) vient de Plutarque; elle transpose le thème de lhomme-microcosme sur le plan social, la société étant assínilée à un grand homme. Mais son enseignement principal est que le Premier citoyen est le gros orteil et que la plèbe constitue la base et les pieds du corps social, rien de plus mais aussi rien de moins. J'ai fait le relevé de tous les passages qui assimilent le peuple de Rome aux Poissons de la mer.

L'odeur de poisson que dégage Caliban est caractéristique du personnage; je rappelle en outre un curieux passage dans lequel Prospero s'exclame:

What I say

my foot my tutor?

(I. ii,.471-472)

devant une tentative de révolte de Ferdinand qu'il a astreint aux corvées manuelles qui sont ordinairement le lot de Caliban.

On trouve dans les propos du peintre, au début de Timon d'Athènes, «The foot above the head» (I. i. 94), pour signifer la non-reconnaissance du vrai mérite. Danchin dans son édition de cette pièce, renvoyait à une pièce de Chapman, The Widow's Tears (II. iv. 168) où Eudora, courtisée brutalement par un inférieur, disait en substance : «Or am I so scant of worthy suitors [...] that I must stoop to make my foot my head" phrase où le sens métaphorique domine : la tête signifiant le maître et foot désignant le domestique.

Je terminerai par quelques mots encore sur La Tempête, qui fut le point de départ de ma recherche. En effet, j'avais depuis longtemps été frappé par le fait que la pièce s'articulait autour de trois personnages très dissemblables, qui représentent en fait trois mondes : Prospero, la magicien, est le Bélier, la tête. Ariel, dont le nom même signifie en hébreu "dieu en forme de lion", est un esprit élémentaire, fait d'air et de feu. Mais son lien avec le ccur apparaît dans le fait qu'il apprend et découvre l'amour humain :

Do you love me, master? No?

Quant à Caliban, il représente les pieds, l'infra-humanité; cependant, à la fin de la pièce, Prospero déclare :

... this thing of darkness I

Acknowledge mine

(V. i. $275-276)$ 
La pièce peut donc être considérée comme un extraordinaire effort pour intégrer les trois mondes, extra-humain, humain, soushumain. Cela confirme bien son caractère testamentaire. Toutefois, Prospero renonce à faire d'Ariel un être presque humain, et il le laisse retourner aux éléments.

Miranda représente la Vierge, et la Sainte Vierge; mais son nom indique aussi qu'elle est la fonction regardante, l'admiration incarnée (elle-même objet d'affection et d'admiration) comme le dit Ferdinand en apprenant son nom :

Admir'd Miranda

Indeed the top of admiration.

(III. i. 37-38)

Or selon Platon (dans le Théétète ou De la science, 155), l'admiration est mère de la sagesse.

Dans Hamlet, comme dans les autres grandes tragédies, les personnages sont répartis dans le zodiaque. La roue aquatique y est ainsi constituée : Cancer : Polonius; Scorpion : la Reine Gertrude; Poissons : Claudius. Le roi est un des deux personnages définis par une notation corporelle, son nom signifiant "boiteux". Une nouvelle fois, le pied désigne un personnage pervers et dangereux.

Chaque fois que les personnages d'une pièce de Shakespeare se trouvent répartis dans le zodiaque par un ensemble d'indications concordantes, cela comporte une double conséquence : d'une part, la pièce constitue, en elle-même, une image du macrocosme, d'autre part, elle représente un corps humain.

Dans les œuvres les plus accomplies, ce corps est, en fait, celui du personnage principal, et les autres rôles sont, en quelque sorte, des fonctions ou des aspects de ce corps et de la personne qui l'occupe. Je crois l'avoir montré déjà dans le cas du Roi Lear. Hamlet et Lear représentent deux versions de l'homme total, s'efforçant de retrouver son origine divine par une remontée des sphères, en prenant appui sur les vicissitudes de l'existence, considérées comme autant d'épreuves probatoires. L'enseignement que véhiculent les deux ouvres dont ils sont les héros associe le meilleur du stoïcisme antique (l'acceptation de soi, l'amor fati) à l'enseignement du Christ; aimer Dieu, aimer son prochain. De là, pour une part, la fascination que ces pièces et ces personnages exercent sur 
nous. Toutefois, sur Hamlet, en dépit des commentaires accumulés, il reste encore à dire, dans la perspective que j'ai esquissée dans ce bref exposé.

JEAN RICHER

Université de Nice

\section{NOTES}

1. James Dauphiné et Jean Richer, Les Structures symboliques du Roi Lear de Shakespeare, Paris, Les Belles Lettres, 1979. J. Richer, Prestiges de la lune et damnation par les étoiles dans le théâtre de Shakespeare, Paris, Les Belles Lettres, 1982 (Le Songe d'une nuit de la mi-été, Roméo et Juliette, Le Marchand de Venise, Macbeth, Othello). Lecture astrologique des pièces romaines de Shakespeare, P. Guy Trédaniel, 1988. (Titus Andronicus, Jules César, Antoine et Cléopâtre, Coriolan).

2. Voir la réimpression préfacée par nous, avec la traduction en anglais de Brian Juden, Images astrologiques des degrés du zodiaque, Nice, Bélisane, 1986.

3. James Dauphiné, Les Structures symboliques dans le thếtre de Shakespeare, Paris, Les Belles Lettres, 1983, p. 200-208.

4. Un peu plus loin dans le même acte, l'indication donnée, légèrement différente, a la même signification: under her breast ... lies a mole (II. iv. 37-38). 